\title{
Giant pleomorphic adenoma of lacrimal gland presenting with diminished vision - a case report
}

\author{
Phani Kumar Mokkapati, ${ }^{1}$ Manisha Mahapatra, ${ }^{2}$ Kandula Satish, ${ }^{3}$ \\ Kodanada Ram ${ }^{4}$ \\ ${ }^{1}$ Department of Neurosurgery; ${ }^{2}$ Department of Pathology; ${ }^{3}$ Department of \\ Ophthalmology; ${ }^{4}$ Department of Surgery, G.S.L General Hospital, Rajahmundry, \\ Andhra Pradesh, India
}

\begin{abstract}
Tumors originating from the lacrimal gland are rare.We present a case of a 40-year-old patient with pleomorphic adenoma of ectopic lacrimal gland in the left orbit. This large-sized tumor was situated deep inside the orbit and compressed the globe and the optic nerve. Patient as a result presented with proptosis and diminshed vision of the left eye (reduced to just perception of light). A total excision of the tumor was accomplished by means of a transcranial approach. The patient recovered well with a good improvement in the visual acuity. A review of the clinical features, radiologic findings, treatment and histopathology is made.
\end{abstract}

Key words: Ectopic lacrimal gland, pleomorphic adenoma, transcranial approach

\section{Introduction}

Lacrimal gland tumors occur relatively rarely accounting for $5-6 \%$ of intraorbital tumors. 'Pleomorphic adenoma is the most common epithelial tumor of the lacrimal gland accounting for $12 \%$ of all lacrimal gland lesions. ${ }^{2}$ These tumors develop in the middle age and have a long slow growing history. They present with proptosis and restriction of ocular movements but do not generally press the globe.

We describe a 40-year-old patient who presented with a large sized pleomorphic adenoma of lacrimal gland manifesting as proptosis and diminished vision owing to compression of the globe.

\section{Case}

A 40-year-old man reported with protrusion of left eye for the past two years. He had dull eye ache and a progressive deterioration in vision for six months. Examination revealed a ten-mm axial proptosis (Fig. 1). There was restriction of ocular movements and vision was reduced to perception of light. Examination of fundus revealed signs of unilateral left sided disc edema likely due to optic nerve compression. T1- and T2-weighted magnetic resonance imaging (MRI) demonstrated an almost iso-intense and slightly heterogenous

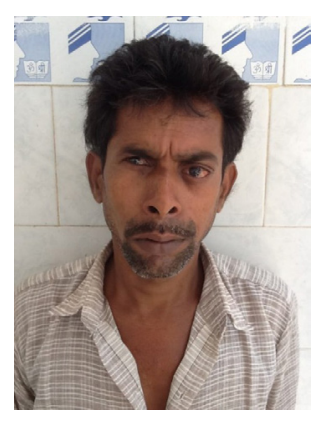

Fig. 1. Patient photograph with left eye proptosis.

Correspondence: Dr. M. Phani Kumar, D.No-54-9-8, Flat- 502, Satya Classic, Addepalli Colony, A.V.A.Road, Rajahmundry-533103, Andhra Pradesh, India 
tumor abutting the orbital roof superiorly, pressing the globe down, having dimensions of $30 \mathrm{~mm} \times 26 \mathrm{~mm} \times 24 \mathrm{~mm}$ (Figs. 2-4).
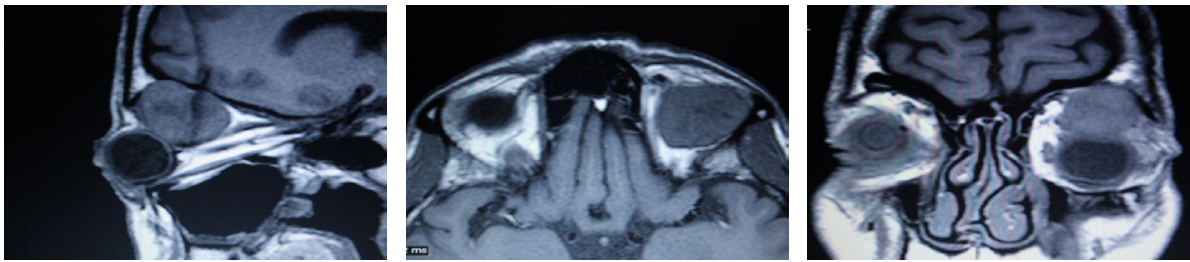

Fig. 2. (left) MRI image (T1 sagittal) showing tumor abutting the left orbital roof and pressing the globe and optic nerve.

Fig. 3. (middle) MRI image (T1 axial) showing tumor in superior part of left orbit.

Fig. 4. (right) MRI image (T1 coronal) showing tumor with extension Onto medial and lateral part of orbit.

Surgery was undertaken under general anesthesia. A combined two-piece frontoorbito-zygomatic craniotomy was made to de-roof the orbital roof and expose the tumor. The tumor was found to be encapsulated, reddish, globular and extraconal in location pressing the globe down. There was no breach of orbital plate or erosion of dura. Total excision of this firm tumor was accomplished with an intact capsule Fig. 5). Normal-looking lacrimal gland was found in fossa lacrimalis.

Histopathological examination of the surgical specimen showed growth of tumor cells as ductal structures with eosinophilic luminal secretions. The ducts were lined by two types of cells, inner cuboidal and outer myoepithelial cells showing vacoulated cytoplasm. Intervening stroma was fibromyxomatous and collagenous at places. The whole picture is indicative of pleomorphic adenoma (Fig. 6).

Postoperatively, vision of the patient improved to $6 / 36$ and proptosis disappeared (Fig. 7).
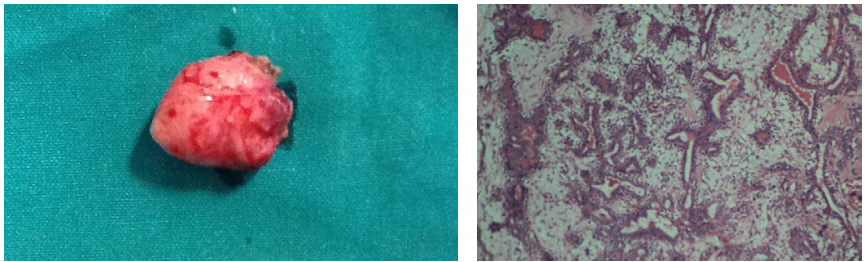

Fig. 5. (left) Excised tumor.

Fig. 6. (middle) Photomicrograph showing numerous ductal structures surrounded by a sheath of myoepithelial cells. Intervening areas show myxomatous change.

Fig. 7. (right) Postoperative photograph of the patient (also seen is the bicoronal incision).

\section{Discussion}

Pleomorphic adenoma is a benign tumor found in the salivary and lacrimal glands. The characteristic histology, an epithelial component and mesenchymal component was first described by Billroth ${ }^{3}$ in 1859, and termed a'mixed tumor' by Minsenn ${ }^{4}$ 
in 1874. Wills ${ }^{5}$ in 1960 coined the name 'pleomorphic adenoma'. Histologically, the tumor is composed of chords of well-differentiated epithelial tubules derived from the ducts of the lacrimal gland within loose myxomatous connective tissue. ${ }^{6}$

The most typical feature of a lacrimal gland neoplasm is proptosis of slow evolution with eventual inferonasal displacement of the globe and limitation of ocular movements. ${ }^{7}$ Diminished vision is not a regular feature as was seen in our case.

The lacrimal gland develops from the basal conjunctival cells as solid buds at eight weeks gestation (20-mm stage). These buds gradually migrate and finally come to lie in the lacrimal gland fossa. The tissue continues to grow after birth and complete differentiation occurs only after three years of age. The accessory lacrimal glands are similarly formed as ectodermal evaginations of the conjunctiva. During this process of development a part of the gland may get sequestered and develop separately, unconnected to the main mass of the lacrimal gland. Should the ectopic tissue lie deep in the orbit it may present as proptosis at any age. ${ }^{7}$

Ectopic lacrimal gland in the conjunctiva and lids is well-known, but in the orbit it is uncommon. Very rarely a pleomorphic adenoma originating in the orbital ectopic gland has been reported. ${ }^{8}$ The location of the tumor in our case suggests origin from ectopic lacrimal gland located in the orbit. Green and Zimmerman ${ }^{9}$ reviewing 35 cases of aberrant lacrimal gland found eight cases in which ectopic lacrimal gland was found deep in the orbit. In all eight cases there was proptosis and they found gland to be showing chronic inflammation in all but two cases. They did not come across mixed tumor of the gland but found hamartomatous malformation in one case and adenocarcinoma associated with ectopic lacrimal gland in another.

Surgery is essential in the management of pleomorphic adenoma of lacrimal glands and type of approach is planned according to the size and location of the tumor. ${ }^{9}$ Successful treatment requires complete removal of the tumor including the capsule. If the capsule is damaged, the myxoid component flows out and the tumor may recur. ${ }^{10}$ In this case, due to the giant size of the tumor and its location in the orbit abutting the orbital roof, a transcranial approach was undertaken by means of a two-piece combined fronto-orbito-zygomatic craniotomy.

\section{Conclusion}

Tumors of the lacrimal gland are rare. Few reports are cited of an ectopic lacrimal gland deep in the orbit. Pleomorphic adenoma arising from these structures has been very rarely reported. A large-sized lesion of similar origin causing compression of the globe and deterioration in vision has hardly been reported. A planned surgical technique aimed at total removal of tumor irrespective of its size with intact capsule is the treatment of choice for achieving an excellent prognosis for patient through improvement in vision and disease free survival.

\section{References}

1. Koike T, Sano S, Tajimas S, Sakaguchi M, Toyode N, Yamamoto Y, et al. A case of pleomorphic adenoma of the lacrimal gland. Nippon Keiseigeka Gakkai Kaishi 1989;9:150-155. 
2. Shields CL, Shields JA, Eagle RC, Ratermell JP. Clinicopathologic review of 142 cases of lacrimal gland lesions. Ophthalmology 1989;96:431-435.

3. Billroth T. Beobachtungen über Geschwülste der Speicheldrüsen. Virchows Arch J Path Anat 1859;17:357-375.

4. Minsenn H, Über Gemischte Geschwülste der Parotis. Göttingen: Universitat Göttingen 1874.

5. Rose GE, Wright JE, Pleomorphic adenoma of the lacrimal gland. Br J Ophthalmol 1992;76:395-400.

6. Shields CL, Shields JA, Eagle RC, RizzoJ. Pleomorphic adenoma of the lacrimal gland. Arch Ophthalmol 1987;105: 560-561.

7. Kaushik NC. Ectopic lacrimal gland in the eyelids. Indian J Ophthalmol 1985;33:65-66.

8. Boparai MS, ChakrabortyM, Sharma RC. Mixed tumor of the lacrimal glands presenting with proptosis and retinal striations. Indian J Ophthalmol 1979;27:49-51.

9. Green WR, Zimmerman LE. Arch Ophthalmol 1967;78,318.

10. Chandrasekhar J, Farr DR, Whear NM. Pleomorphic adenoma of the lacrimal gland: case report. $\mathrm{Br} J$ Oral Maxillofac Surg 2001;39:390-393.

11. Becelli R, Carboni A, Cassoni A, Renzi G, Jannetti G. Pleomorphic adenoma of the lacrimal gland: presentation of a clinical case of relapse. J Craniofac Surg 2002;13:49-52. 